SangHyun LEE

\title{
Applying system dynamics to strategic decision making in construction
}

(C) The Author(s) 2017. Published by Higher Education Press. This is an open access article under the CC BY license (http:// creativecommons.org/licenses/by/4.0)

\begin{abstract}
The author discusses the application of System Dynamics to high-level strategic simulation in construction. In particular, System Dynamics' strength on representing feedback processes, aggregation, soft variables, and continuous simulation clock for high-level simulation are discussed using real modeling examples. From this exercise, it is concluded that System Dynamics offers a great potential for strategic simulation in construction. Further, the author proposes a comprehensive simulation framework that integrates System Dynamics and Discrete Event Simulation for a strategic decision making process in construction where operational details should be taken into account.
\end{abstract}

Keywords strategic project management, construction management, system dynamics, feedback process, hybrid simulation

\section{Introduction}

Computer simulation has been widely applied for diverse construction decision-making processes by enabling 'what-if' scenarios. Discrete Event Simulation (DES) has been a primary means for such simulation, representing construction operational details. Taking into account the similarities between construction operations and queuing theory, DES that represents queues well would be a proper means to represent operations details in construction.

However, there is a strong need to apply computer simulation to high-level strategic decision making. Based

Received January 10, 2017; accepted February 16, 2017

SangHyun LEE ( $\bowtie)$

Associate Professor and John L. Tishman Faculty Scholar, Tishman Construction Management Program, Department of Civil and Environmental Engineering, University of Michigan, Ann Arbor, MI 48109, USA

E-mail: shdpm@umich.edu on the analysis of 3500 projects, Morris and Hough (1987) reported that a lack of strategic decision-making and analysis is a major cause for the failure of many of these projects. Taking into consideration the complex relationships among processes, subcontractors, resources, etc. in a construction project, obtaining closed form solutions and determining appropriate policies are difficult and thus, simulation can be a prominent means to address this complex situation. Despite its need, applying simulation to a high-level strategic decision making process requires a holistic approach because appropriate policies cannot be made without an understanding of a comprehensive grasp of a construction project. As such, DES models, based on reductionism and randomness, are often difficult to be used to make managerial decisions and a policies (Martin and Raffo, 2001).

In an effort to address this issue, the author has been applying System Dynamics (SD) to the strategic decision making process in construction as complementary to DES. SD was developed in the late 1950s to pply control theory to the analysis of industrial systems (Richardson, 1985). It has been widely applied to complex industrial, economic, social, and environmental systems of all kinds (Turek, 1995). In particular, SD's emphasis on system structure can greatly contribute to the strategic decision making process identifying how system structure generates dynamic and complex behavior over time (e.g., during actual execution of a project).

In this paper, the author discusses several issues raised from the application of SD to real-world construction projects. Specifically, the model structure, the level of aggregation, soft variables, and simulation clock in strategic simulation are extensively discussed because they facilitate strategic decision making. In addition, the nature and extent of contributions to the high-level strategic decision making process that can be made by $\mathrm{SD}$ are explored throughout the paper with real modeling examples. Last but not least, hybrid SD and DES is proposed as a comprehensive simulation framework when 
operational details are an important element for strategic decision making.

\section{Feedback processes}

Construction projects are inherently complex and dynamic, involving multiple feedback processes (Sterman, 1992). Such feedback processes usually drive the complexity of construction projects. There are only two types of feedback processes in SD: reinforcing and balancing (Sterman, 2000). The dynamics of a system (e.g., construction project) results from the continued interaction of these two types of feedback processes among the components of the system, not from the complexity of the components themselves (Sterman, 2000). Understanding these feedback processes and their interactions is particularly important in strategic decision making because good policy decisions cannot come without a full grasp of the system.

A main idea behind SD is that system structure causes its dynamic behaviors (e.g., changes over time) (Richardson, 1985). Compared with DES's emphasis on input randomness (e.g., input modeling), SD's emphasis on the system structure facilitates a better understanding of the system. For instance, in one of the case projects where the author applied SD, a project manager adopted overtime to accelerate project progress to remedy behind schedule. $\mathrm{He} / \mathrm{she}$ believed that the delays could be reversed by increasing work hours. Though the early results of implementing overtime did suggest that the schedule was being accelerated, it soon became clear that this was not the case any longer.

This situation can be explained by SD's well-known feedback processes related to overtime. As denoted by Loop A (i.e., balancing feedback) in Fig. 1, the adoption of overtime means the increase of work hours. As work hours are increased, production (rate) increases, which deceases the amount of work to do. Consequently, the decreased work to do will result in the reduction of work hours at the point when there is no need to accelerate the schedule any more (e.g., catching up behind schedule). This balancing loop shows a positive impact of overtime. However, if work hours increase, workers' fatigue increases, which in turn, results in a decrease in production (rate). To compensate for this decrease in production (rate), more work hours are required. This loss in production (rate) is the unexpected result of the adoption of overtime, denoted by Loop B (i.e., reinforcing feedback) in Fig. 1.

This identification of the system structure on overtime enables the development of better policies in project management. For example, when overtime is taken into account, workers' fatigue need be taken into account as well if overtime is expected for a long time. Previous studies indicate that prolonged work hours begin to reduce productivity after a week or two, with the full effect requiring a somewhat longer duration to develop (Oliva, 1996). Thus, if the situation requires overtime for a long time, other policies such as hiring new workers could be considered. As demonstrated by this example, an understanding of the feedback processes and their interactions is a significant premise for appropriate policies. In this sense, SD's emphasis on feedback processes is a great asset to aid the strategic decision making process in project management (Williams, 2002).

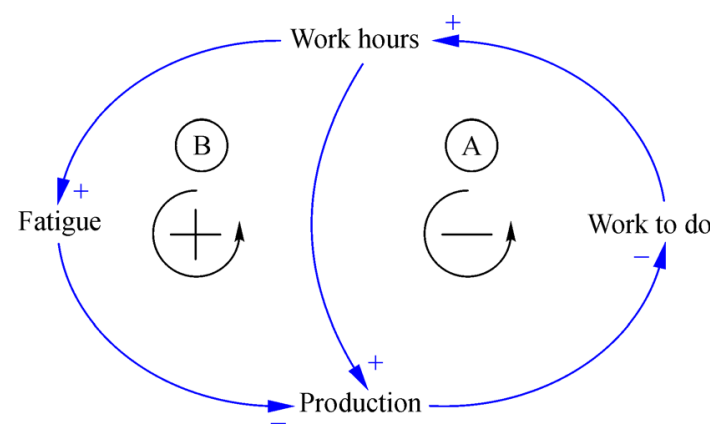

Fig. 1 Feedback processes related to overtime

\section{Aggregation}

When a simulation model is developed for high-level strategic decision making (i.e., policy making), modeling the whole world in detail is not necessary. Rather, if the model can accurately represent and explain success or failure of a policy of interest, it is more than enough. After this, the model can be extended to incorporate more policyrelated components if it is a user's interest and need. Such a step-by-step process (not modeling the whole world at once) is much related to the model's economics since building the whole world model requires a lot of efforts. In this sense, aggregation is an important aspect in high-level strategic simulation.

One of the features of SD is its ability of aggregate representation of the world. For instance, stock and flow structure, which is a core model structure in SD, is based on aggregation. Stock and flow structure characterizes the state of the system and generates information upon which decision and actions are based by representing the system inertia and memory (Sterman, 2000). Stocks (e.g., rectangles in Fig. 2) represent stored quantities, and flows (e.g., arrows in Fig. 2) represent control quantities flowing into and out of stocks (Peña-Mora and Park, 2001).

Figure 2 shows stock and flow structure for 'work execution with errors' in construction (Lee and Peña-Mora 2007). Before execution, all tasks are in the stock of 'Work To Do' (A in Fig. 2). Based on the flow of 'Work Rate' (B in Fig. 2), determined by the number of workers and their productivity, tasks in 'Work To Do' move into the stock of 'Work Awaiting QM' (C in Fig. 2), meaning that tasks are done and are ready for quality management (e.g., 


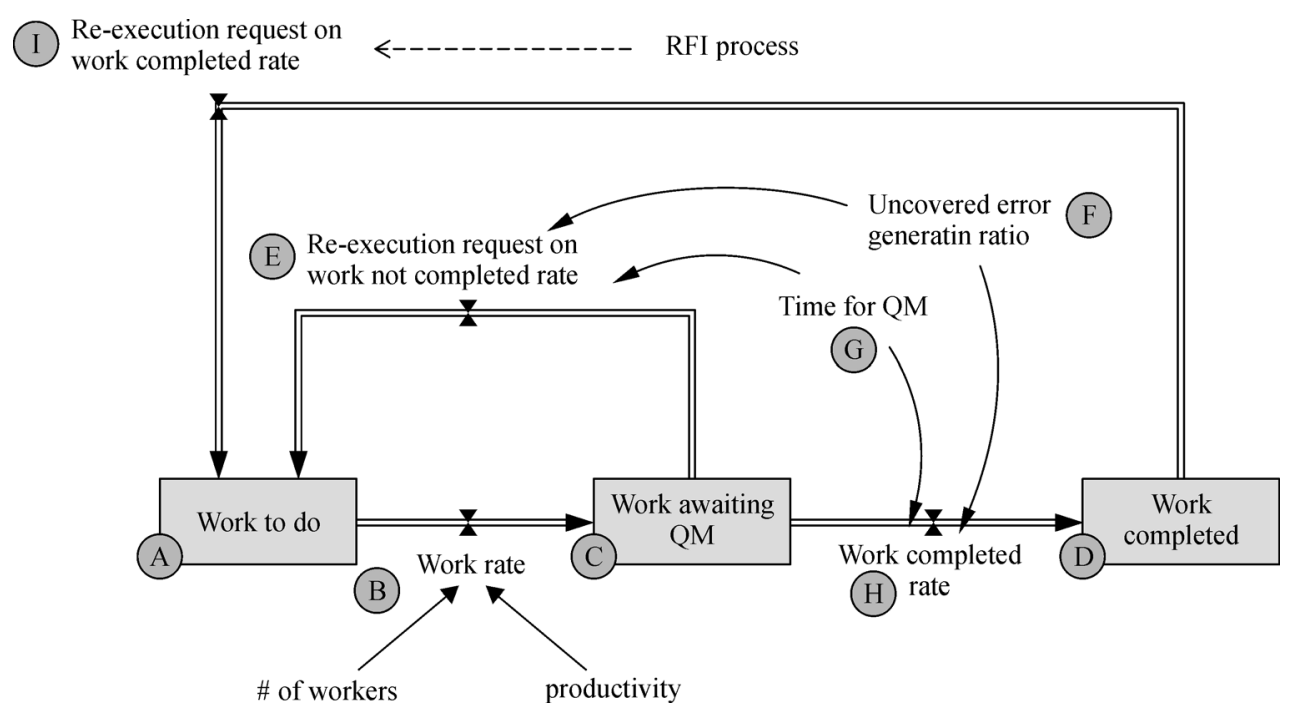

Fig. 2 Stock and flow structure for work execution with errors

inspection). Here, tasks are assumed as homogenous. In reality, however, they are not homogenous in general. For example, each task can require a different number of people and productivity. However, SD does not have to focus on such details at this stage because the focus is the aggregate representation of the world in order for us to understand the world analytically.

Continuing with this model structure, if the work done passes quality management, it can move to the stock of 'Work Completed' (D in Fig. 2), otherwise, it has to be performed again due to uncovered errors. Thus, the flow of 'Re-Execution Request on Work Not Completed Rate' (E in Fig. 2) would be determined by 'Uncovered Error Generation Ratio' ( $F$ in Fig. 2), the probability of uncovering errors, 'Time For Quality Management' (G in Fig. 2), and average time taken for quality management. However, passing quality management does not ensure that there are no errors because errors can be hidden if the quality management process is imperfect. This means that the flow of 'Work Completed Rate' (H in Fig. 2) can contain hidden errors, which would be determined by the 'Hidden Error Generation Ratio' (i.e., one minus 'Uncovered Error Generation Ratio'). Thus, the stock of 'Work Completed' will have hidden errors. These hidden errors will be addressed at a later stage of the project with the 'Request For Information' (RFI) process (i.e., flow of 'ReExecution Request on Work Completed Rate' - I in Fig. 2), which is related to another model structure. The detailed formulations are included in the Appendix.

This aggregate representation may not be adequate for detailed analysis of construction operations. However, the overall representation of work execution with errors suggests that if hidden errors need to be reduced, attention should be paid to the improvement of quality management (e.g., improving Uncovered Error Generation Ratio - F in
Fig. 2) and/or the RFI process (e.g., reducing the time to be taken for RFI - I in Fig. 2). Further, simulation results can contribute to the decision making process showing the extent of the benefits that could be obtained. For example, simulation results in the case project showed that a decrease in RFI response time could reduce the project completion time as much as $4.86 \%$ (Lee et al., 2005).

As demonstrated in this modeling example, SD's aggregate representation can contribute to an understanding of the overall system so informed strategic decisions can be made. Once an overall understanding is developed, a detailed decision can be supported by adjusting the level of aggregation in SD.

\section{Soft variables}

In most construction simulation, the majority of variables are 'hard' variables that are available as quantitative metrics and numerical data. However, most of what we know about the world (e.g., construction project) is descriptive, impressionistic, and has never been recorded (Sterman, 2000). For example, though the fatigue shown in Fig. 1 has rarely been used in construction simulation, it certainly plays a crucial role in workers' productivity. Thus, 'soft' variables, such as goals, perceptions, and expectations, are significant in representing the world. Particularly, in strategic construction simulation, soft variables become more important because some policies are derived from these soft variables. That's why SD encourages the use of soft variables in the strategic decision making process. Suppose overtime can be allowed when the project is behind schedule. In this case, the issue would be how the project manager should actually implement the overtime policy. Figure 3 shows a 


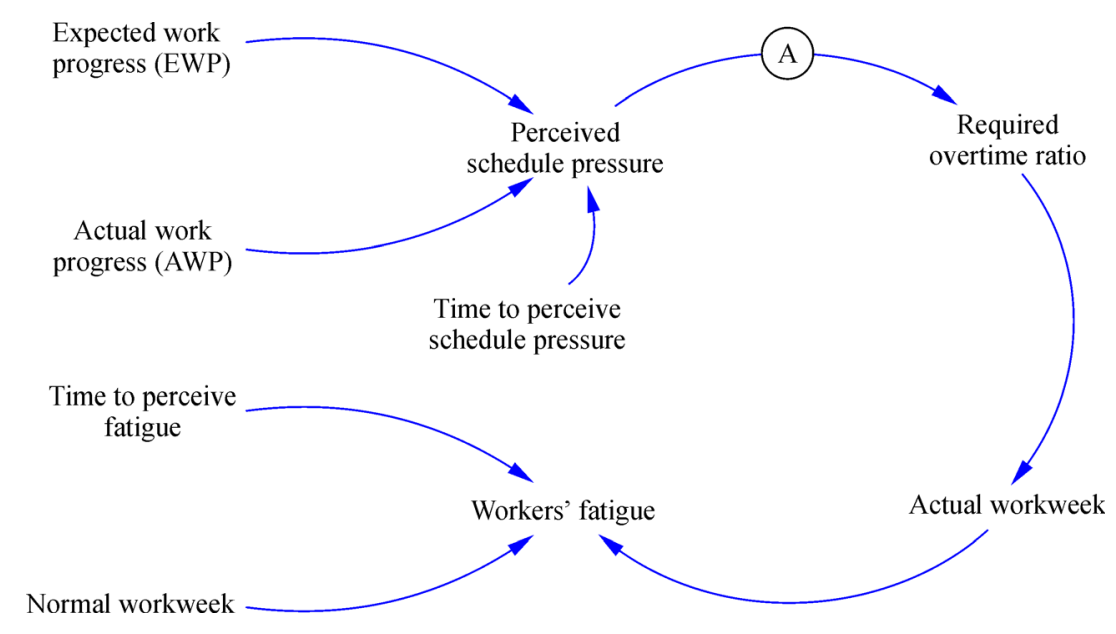

Fig. 3 Modeling how the policy can be triggered and affect performance using soft variables

simplified SD model that captures how this can be triggered. In the manager's mental model, there is Expected Work Progress (EWP) derived from the estimated work scope and the expected productivity. In addition, Actual Work Progress (AWP) will be reported through the monitoring process. When AWP is not reaching EWP, and the gap between AWP and EWP exceeds a certain threshold, the project manager perceives the schedule pressure with a time delay corresponding to the triggering threshold. This is the moment when the manager triggers an overtime policy, if allowed (A in Fig. 3). Further, the degree of overtime (i.e., overtime ratio) and corresponding work hours will be determined based off the perceived schedule pressure. For example, if a normal workweek (i.e., working hours per week) is $40 \mathrm{~h}$ per week and we assume that the overtime ratio ranges from 1.0 to 1.3 , workers can work up to $52 \mathrm{~h}$ per week in response to increasing perceived schedule pressure. Thus, an actual workweek can be calculated during the adoption of overtime and consequently, workers' fatigue can be derived by comparing an actual workweek to a normal workweek.

Likewise, the wide use of soft variables in SD makes us understand how a new policy can be implemented and further, how it can affect construction performance, which in turn, contributes to determining a good policy.

\section{Simulation clock}

Simulation clock is another important issue in strategic simulation. DES models recalculate their state variables only at a discrete set of points, referred to as 'event times' (Banks et al., 2001) assuming that nothing happens in the intervals between event times. As such, intervals usually have an inconsistent step size, and this can cause unrealistic representations in strategic simulation (Lee et al., 2009).
Continuing with the previous overtime example, assume that a manager made a policy that overtime would be adopted when the overtime ratio is 1.2 (i.e., schedule pressure is 1.2 when AWP is behind EWP as much as $20 \%$ if we exclude the effect of a time delay). In DES models, since the overtime ratio is only recalculated at each event time, such a managerial action (or policy) can only be adopted at the event times. As shown in Fig. 4, the planned managerial action (A in Fig. 4) will not be triggered because there exists no event time at the exact moment when the overtime ratio reaches the threshold (i.e., 1.2). Instead, the managerial action will be triggered at Event 3, when the overtime ratio has exceeded 1.2 (B in Fig. 4). As a result, the managerial action is taken later than it should have been. This implies that there can be work hour shortage due to the late introduction of more working hours. As a result, inconsistent step size will make managerial actions (or policies) less reliable.

On the other hand, SD models recalculate variable states with consistent time intervals (i.e., continuous simulation) that the user chose and thus, could be more responsive to a change in the project environment than DES models by decreasing time step size. In the case project, several policies are implemented to analyze their impact on construction performance, such as hiring new workers and adopting overtime. The use of a small and consistent time step size avoided the unrealistic representations of triggering policies that may happen in DES models.

\section{Conclusions}

The author discussed four major issues concerning the application of System Dynamics to high-level strategic simulation in construction. Based on the modeling examples based on the real-world projects, the author concluded that SD can greatly contribute to the strategic decision making process supporting: (1) the dynamic 


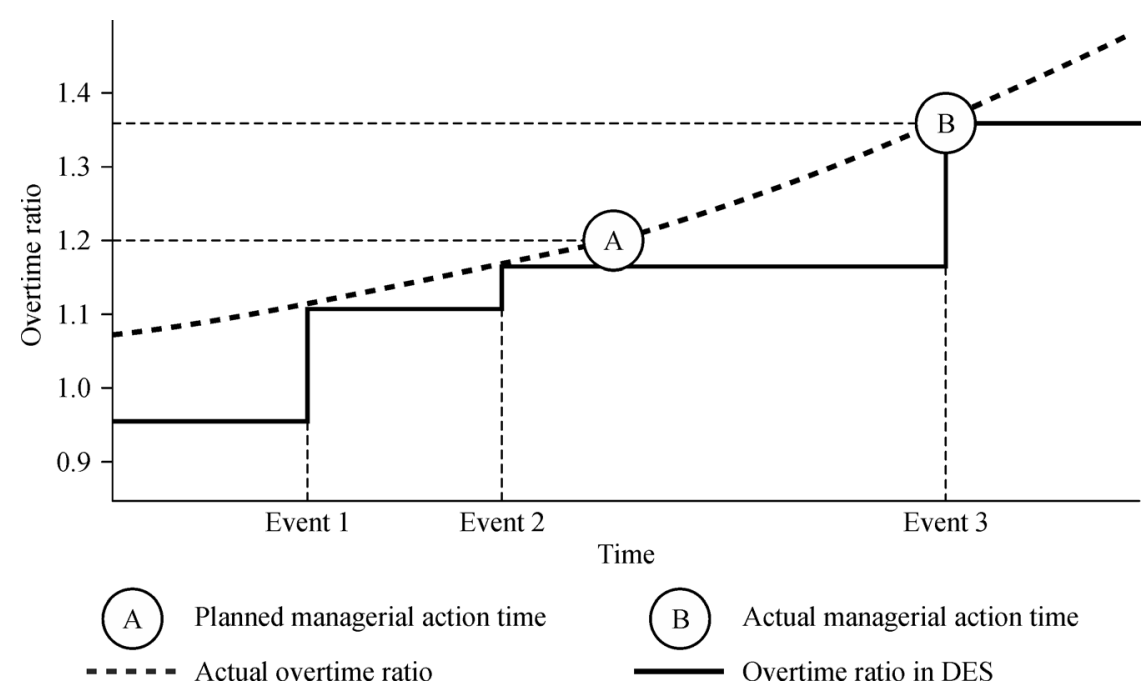

Fig. 4 Inconsistent time step in DES

behavior generated from feedback processes related to policies; (2) aggregate representation to understand the overall system; (3) soft variables that explain how policies can be triggered and affect performance; and (4) consistent and small time step size (i.e., continuous simulation) to capture the moment that policies are triggered in a realistic manner.

However, the strategic decision making process often needs to be aligned with operational analysis. Though the strategic analysis with SD alone may provide valuable insights to a manager, it is difficult to apply these insights to operations in detail (Lee et al., 2009). In addition, inconsistency between strategic and operational analyses can be another major reason for the project failures (Callahan and Brooks, 2004). In this context, the author proposes hybrid SD and DES as an alternative capable of incorporating DES's ability to capture construction operational details to SD's high level system representation. Thus, the hybrid simulation aims to support both the strategic and the operational aspects of a construction project that cannot be fully represented by either a SD or DES model alone. An interested reader is referred to the author's related papers that discuss the application of hybrid SD and DES to construction (Alvanchi et al., 2011; Hwang et al., 2016; Lee et al., 2009).

\section{Appendix}

1. Work to do $=$ INTEGRAL (Re-execution request on work not completed rate + Re-execution request on work completed rate - Work rate)

2. Work awaiting QM = INTEGRAL (Work rate - work completion rate - Re-execution request on work not completed rate)

3. Work completed $=$ INTEGRAL (Work completed rate
- Re-execution request on work completed rate)

4. Work rate $=\#$ of workers $*$ Worker's productivity

5. Work completed rate $=\{$ Work awaiting $\mathrm{QM} *(1-$ uncovered error generation ratio) $\}$ / Time for quality management

6. Re-execution request on work not completed rate $=$ (Work awaiting QM * Uncovered error generation ratio) / Time for quality management

\section{References}

Alvanchi A, Lee S, AbouRizk S (2011). Modeling framework and architecture for hybrid system dynamics and discrete event simulation for construction. Computer-Aided Civil and Infrastructure Engineering, 26(2): 77-91

Banks J, Carson J S, Nelson B L, Nicol D M (2001). Discrete-Event System Simulation. 3rd ed.

Callahan K, Brooks L (2004). Essentials of strategic project management. Project Management, 39(1): 95

Hwang S., Park M., Lee H (2016). Hybrid simulation framework for immediate facility restoration planning after a catastrophic disaster. Journal of Construction Engineering and Management, 142(8): 04016026

Lee S, Han S, Peña-Mora F (2009). Integrating construction operation and context in large-scale construction using hybrid computer simulation. Journal of Computing in Civil Engineering, 23(2): 75-83

Lee S, Peña-Mora F (2007). Understanding iterative error and change cycles in large-scale design and construction. System Dynamics Review, 23(1): 35-60

Lee S, Peña-Mora F, Park M (2005). Quality and change management model for large scale concurrent design and construction projects. Journal of Construction Engineering and Management, 131(8): 890902

Martin R, Raffo D (2001). Application of a hybrid process simulation model to a software development project. Journal of Systems and 
Software, 59(3): 237-246

Morris P, Hough G (1987). The anatomy of major projects - A study of the reality of project management. Hoboken: John Wiley \& Sons

Oliva R (1996). A Dynamic theory of service delivery: implication for managing service quality. Dissertation for the Doctoral Degree. Cambridge: Massachusetts Institute of Technology

Peña-Mora F, Li M (2001). Dynamic planning and control methodology for design/build fast-track construction projects. Journal of Construction Engineering and Management, 127(1): 1-17

Peña-Mora F, Park M (2001). Dynamic planning for fast-tracking building construction projects. Journal of Construction Engineering and Management, 127(6): 445-456
Richardson G (1985). Introduction to the System Dynamics Review. System Dynamics Review, Albany, NY, 1(1): 1-3

Sterman J (2000). Business dynamics: system thinking and modeling for a complex world. McGraw-Hill Companies, New York, NY

Sterman J D (1992). System dynamics modeling for project management. http://jsterman.scripts.mit.edu/docs/Sterman-1992-SystemDynamicsModeling.pdf, 2016-12-20

Turek M (1995). System dynamics analysis of financial factors in nuclear power plant operations. Dissertation for the Master Degree.Cambridge: Massachusetts Institute of Technology

Williams T (2002). Modeling complex projects. Hoboken: John Wiley \& Sons 